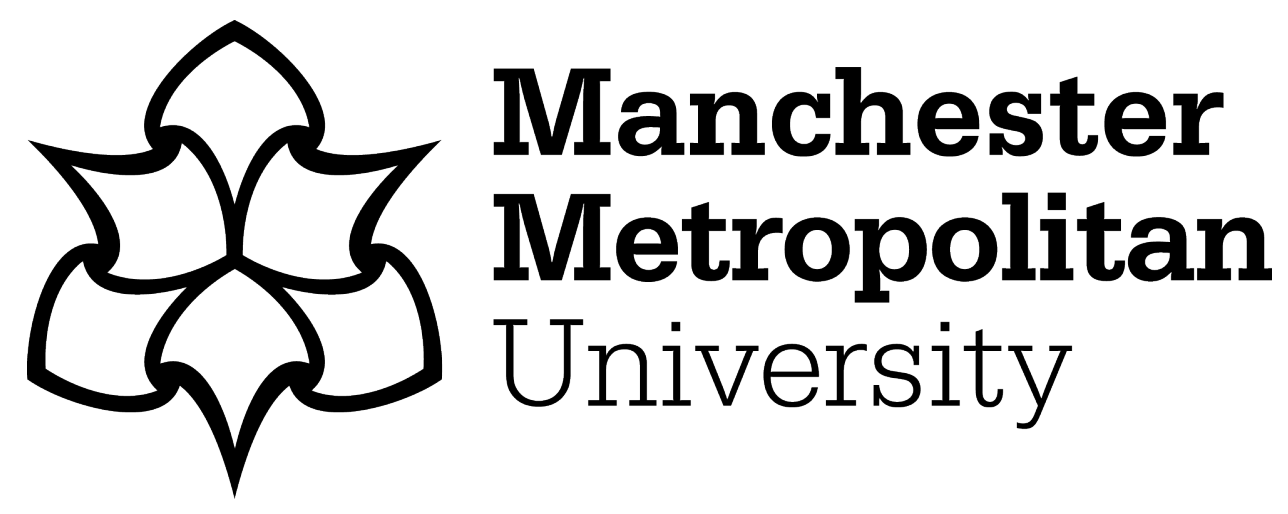

Edwards, Benjamin (2015) Open Archaeology: Definitions, Challenges and Context. In: Open Source Archaeology: Ethics and Practice. De Gruyter, pp. 1-5. ISBN 9783110440171

Downloaded from: https://e-space.mmu.ac.uk/619568/

Version: Published Version

Publisher: De Gruyter

DOI: https://doi.org/10.1515/9783110440171

Please cite the published version 


\section{Open Archaeology: Definitions, Challenges and Context}

\subsection{Introduction}

Over the last two decades archaeology has slowly been going through an information revolution, affecting the ways in which it is researched and published. These changes have come about as a result of an idea: being 'open'. Open source software, open access to archaeological data and open ethics. 'Open' has become an increasingly attractive thing to be; from research, to corporations and governments. Openness gives an air of transparency, ideas of public accountability and scientific repeatability, and as such provides a buzzword for perceived public good (Costa et al., 2014; Lake, 2012). In this volume, the term 'open' is given a specific definition:

"A piece of content or data is open if anyone is free to use, reuse, and redistribute it - subject only, at most, to the requirement to attribute and/or share-alike.” (Open Definition)

\section{2 'Open Source’ Archaeology and 'Open’ Archaeology}

Although based on the same ideas of openness, open source archaeology and open archaeology have come to mean very different things. Open source archaeology comes from the open source software example of the computer sciences; whereas open archaeology emerges from the concepts of open publishing and free access to archaeological datasets.

\subsection{Open Source Archaeology}

'Open-source software' is a term used to describe computer programs that are distributed as readable program source code - statements written in a (high-level) programming language. This availability of the source codes allows the end user to not only run the final program but manipulate, change, redevelop and understand how the underlying functionality of the program works. FOSS, free and open-source software, is not just the software itself but also a repository of knowledge for the tool (Ducke 2012 and see Ducke this volume: Chapter 7).

Benjamin Edwards: Manchester Metropolitan University, Manchester, UK Andrew T. Wilson: Bangor University, Bangor, UK 


\subsection{Open Archaeology}

Open archaeology, in contrast, is focused on ensuring datasets and publications are freely available for use by the wider academic community and the public. Data and publication are two related but distinct strands in this movement, though both are now coming to wider attention. Open publication (see below) has been actively placed on the agenda by both national and European governments in recent years, with public policy now backing the idea that publically funded research should be freely available to the public, although this is currently limited to journal articles and conference proceedings. Open data is at the same time a very old fashioned but also radical idea. It has long been a principle of the natural sciences that experiments should be reproducible, and that datasets should therefore be available to other researchers. The radical element here, and that which is contributing to the 'open' movement, is the nature of this access. It has been a slow start, but databases are now becoming available online in raw and unprocessed form, be these statistical, excavation archive, GIS-based survey or image/3D data archives.

As examples, services such as the UK Archaeology Data Service, Open Context and the Digital Archaeological Record have been pioneering the sharing of archaeological data via the internet, with licenses that encourage re-use. Private bodies such as Oxford Archaeology and Wessex Archaeology have started to also make their grey literature available as an open archive (Costa et al., 2014). This breaks away from the model of data storage, where it is nominally accessible and in reality subject to controlled release by individuals or research organisations. Now the data is becoming accessible. However, whilst the movement is gathering pace, it is still rare to see published datasets alongside finished articles, and also rare to be given a dataset required to reproduce an analysis and produce results. Thus it is possible to define two distinction models of data sharing: dynamic datasets that continue to be updated, versus static datasets that are released once as a finished resource.

\subsection{The Public Context of Open Access}

The move towards open software and open archaeology is not occurring in a vacuum. Open-access is a trend that cuts across disciplinary boundaries, and is also finding support in political and policy-making spheres, reflected in the priorities of research funding bodies. In the UK, HEFCE (the Higher Education Funding Council for England), the AHRC (Arts and Humanities Research Council), and the ESRC (Economic and Social Research Council) have recently published a new policy on open access to scholarly research. This states, amongst other things, that the content of all peerreviewed journal articles and conference proceedings must be made available as open access through institutional repositories once an embargo period has elapsed, with implications for further funding eligibility if this requirement is not met (Higher Ed- 
ucation Funding Council for England, 2014). Whilst this policy does not yet apply to monographs or data, it surely illuminates a trend that will only continue to gather momentum in coming years, and one for which the academic community must be prepared.

This output-based priority for open access is also reflected in project-design phase in the form of requirements placed on new projects in the arts and social sciences. It is increasingly common to find funding calls from major European research bodies placing an emphasis on public engagement and access to data. Ring-fenced funding calls, such as the AHRC's 'Connected Communities' programme or the European 'Horizon 2020' scheme, stressing the connections between academic research and public access, are aimed at bridging the gap between the production of academic knowledge and its impact in society - open-access to both data and interpretation are seen as key to success in these programmes. Similarly, the move toward measuring the 'impact' of academic research is relevant in this regard. Whilst open access is not the only tool to ensure 'impact' (defined as the extent to which the results of research make a difference to society, culture or policy-making outside of the academy), it is seen as an important part of any strategy that attempts to engage the wider world in the practice or results of research. How open access contributes to measures of impact differs across the sector, but can include the direct public participation in data collection through to the public availability of research outputs in a manner that his engaging and aimed at a non-specialist audience.

This movement toward open access has not been entirely philanthropically motivated however. Whilst individual researchers are clearly committed to the ideals of open research and open access, as the content of this volume testifies, it is certainly true that the public mood toward academic research is also changing. This has found its expression in the UK recently, with the government's response to Finch Group report (BIS 2012), a report into open access in UK academia by Dame Janet Finch, recommended the removal of paywalls surrounding published academic research that was funded by the taxpayer through the UK research councils. Universities will now be expected to pay the costs of open access up front. Unsurprisingly, this move was illustrative of wider international trends, with 2012 seeing the European Research Council setting out a new policy on open access to research. Research funded by the ERC must now be made available as open access within six months of its publications date (European Research Council, 2012).

On a broader socio-cultural level, it is possible that these policy-based movements in open access are reflecting trends with other roots. In the UK, recent economic difficulties have either prompted, or been used as an excuse for (the choice here is left to the discretion of the reader), changes to the way in which University courses are funded. Heritage and archaeology have suffered alongside other social sciences because they do not fit the 'STEM' agenda of science, technology, engineering and maths', losing government subsidy as a result. The humanities and social sciences find themselves in the (some would say) ridiculous position of being forced to justify 
their own existence, with the additional difficulty that the 'debate' is framed in a utilitarian language that presupposes the greater importance of the hard sciences. 'Impact' in this context becomes one of the measures, either pernicious or otherwise, of this justification of existence. Whether one agrees with these changes in policy and attitude toward the social sciences is not strictly relevant for this volume. What is clear, however, is that in the new academic, financial and socio-cultural environment that researchers find themselves, open access to research is now not only a moral imperative - it is increasingly vital for the survival of meaningfully funded research.

\subsection{Open Ethics}

With the development of 'open' access within archaeology, a new set of open ethical issues surrounding the use and distribution of the data have come to light. These focus on the types of data that are made 'open' and, critically, its quality. Unpublished research is a key area where open data may have a transformative impact, but also an area where ethical considerations become of relevance. There are various reasons for non-publication, but the sheer cost of bringing archaeological research (especially fieldwork) to formal publication is often a key issue, and there is a significant backlog of mid-twentieth century excavation unpublished in the UK alone. In such cases, we know that some data exists, even if in a raw and unprocessed form (most likely a paper archive). While some might argue that publishing such data without offering a synthetic overview alongside would make for a very limited resource, it is undoubtedly better to have access to data than to have nothing at all. It seems that the greater evil would be to allow such data to remain utterly unpublished, given the relatively low cost of photographically digitising paper archives.

Opening up of grey data (i.e. that collected and published as part of commercial archaeology, usually associated with the planning process, see Huggett, this volume: Chapter 2) is in the view of many, as a way to meet the minimal requirements to publish research (Costa et al., 2014). If these publications, as part of these requirements, had to include the raw research data this would be major step forward for open data. The current view is that a publication is more of a report on the research rather than the total outcome from archaeological activity, as such presented with limited interpretation and, lacking all but the presented data. Reasons for this disjunction are varied, and depend on national context, but a major problem is the cost of making data available (both via publication and permanent online resources) for commercial companies involved in producing grey literature, who work on tight budgets and cannot justify non-statutory expenses. We need to transform our understanding of what constitutes full and satisfactory publication, but accept that this will come with an attached cost. This is one of the great challenges of the open data movement - how to involve commercial data producers. What is certain, is that this involvement should be an ethical imperative (Costa et al., 2014). 


\subsection{Outline of the Volume}

This volume came about because of a perceived lack of published works on the ideas surrounding 'open' archaeology. The transformation to open access in archaeological data has not been examined in sufficient detail. Through a series of papers this volume sets out to examine, not only the open archaeological software currently being employed and open access to archaeological information, but also the emerging change in culture and ethics this 'open' revolution is producing. As such, this volume has three mains themes throughout; open source software, open archaeological data, and open ethics. Each paper touches on at least two or all of these themes, and as such they are hard to pigeonhole. What this volume also demonstrates is the breadth of work being undertaken with an open ethic, and the commitment of individuals and teams of researchers driven by personal belief to be at the forefront of an emerging field, actively creating and shaping a vision of open archaeology that will be an important legacy in the future.

\section{Bibliography}

Costa, S., Beck, A., Bevan, A. and Ogden, J. (2014), Defining and advocating open data in archaeology, in 'Archaeology in the Digital Era: Proceedings of the 40th Annual Conference of Computer Applications and Quantitative Methods in Archaeology'.

Ducke, B. (2012), 'Natives of a connected world: free and open source software in archaeology', World Archaeology 44(4), 571-579.

European Research Council (2012), 'European research council open access guidelines for researchers'.

URL: http://erc.europa.eu/sites/default/files/document/file/open_access_policy_researchers_ funded_ERC.pdf

Higher Education Funding Council for England (2014), 'STEM teaching capital funding allocation'. URL: http://www.hefce.ac.uk/pubs/year/2014/cl022014/name, 85551, en.html

Lake, M. (2012), ‘Open archaeology’, World Archaeology 44(4), 471-478. 